\title{
Hormone \\ replacement therapy and cataract: a population-based case-control study
}

FO Aina' ${ }^{1}$, L Smeeth', R Hubbard², LS Hurt ${ }^{3}$ and AE Fletcher ${ }^{1}$

\begin{abstract}
Purpose Laboratory studies have suggested that hormone replacement therapy (HRT) may protect against the development of cataract, but epidemiological studies in humans have thus far been inconclusive. The aim of this study was to assess the association between hormone replacement therapy and cataract. Methods Population-based case-control study using data from the General Practice Research Database in the UK. Participants were 10000 women aged 45 years and over with diagnosed cataract and 10000 controls matched on age, general practice, and calendar period.
\end{abstract}

Results The crude odds ratio for the association between cataract and ever-use of oestrogen-only hormone replacement therapy was 1.13 (95\% CI 0.99-1.29). This reduced to 0.81 (95\% CI 0.71-0.94) after adjustment for consultation rate. Similarly, the crude odds ratio for the association between cataract and ever-use of a formulation containing oestrogen and progestogen was 1.18 (95\% CI 1.01-1.39), reducing to $0.86(95 \% \mathrm{CI} 0.72-1.02)$ after adjustment for consultation rate.

Conclusions Oestrogen-only and oestrogenprogestogen hormone replacement therapies are associated with a small reduced risk of cataract. This data adds to the growing body of evidence on the effects of HRT on health. All potential benefits and risks of this therapy should be taken into account when considering its use. Eye (2006) 20, 417-422. doi:10.1038/sj.eye.6701877; published online 22 April 2005

Keywords: cataract; hormone replacement therapy; case-control

\section{Introduction}

Hormone replacement therapy (HRT) is one of the most widely prescribed drugs worldwide, with over 20 million women using it in some form in the late 1990s. ${ }^{1}$ However, it is a drug beset by controversy. Recent studies have shown consistent evidence of an increased risk in incident and fatal breast cancer with longterm use, ${ }^{2,3}$ and previous studies suggesting a protective effect on cardiovascular disease have not been confirmed. ${ }^{2}$ Nevertheless, HRT has its benefits, most notably in the treatment of menopausal symptoms. ${ }^{4}$ It is also useful for the prevention of osteoporosis and reduces fractures in postmenopausal women. ${ }^{5}$ In addition, evidence from laboratory studies has suggested that it may protect against the development of cataract. ${ }^{6,7}$

Cataract is a major cause of poor vision and blindness, ${ }^{8}$ with the worldwide prevalence estimated to increase from 16 million cases in 1997 to 40 million by the year $2020 .{ }^{9}$ Any factor that reduces the incidence of cataract may thus be of great public health significance. Despite evidence from animal studies, previous epidemiological studies of the association between HRT and cataract have produced inconclusive results. ${ }^{10-17}$ We therefore conducted a case-control study to examine whether the use of HRT was associated with a reduction in cataract diagnoses, using data from the United Kingdom General Practice Research Database.

\section{Patients and methods}

The General Practice Research Database (GPRD) is the largest longitudinal clinical research database in the UK. It was set up in 1987 and is now managed by the Medicines and Healthcare Products Regulatory Agency. It consists of complete prescribing and diagnostic information on patients registered with general practices that are broadly representative of all general practices in the UK in size and
${ }^{1}$ Department of Epidemiology and Population Health, London School of Hygiene and Tropical Medicine, Keppel Street, London, UK

${ }^{2}$ Division of Epidemiology and Public Health, University of Nottingham, Clinical Sciences Building, Nottingham City Hospital, UK

\footnotetext{
${ }^{3}$ Infectious Disease Epidemiology Unit, London School of Hygiene and Tropical Medicine, Keppel Street,

London, UK
}

Correspondence: L Smeeth Department of Epidemiology and Population Health, London School of Hygiene and Tropical Medicine, Keppel Street, London WC1E 7HT, UK Tel: + 442079272296 ; Fax: + 442075806897 . E-mail: liam.smeeth@ Ishtm.ac.uk

Received: 27 July 2004 Accepted: 20 October 2004 Published online: 22 April 2005

Conflict of interest: None. 
geographical distribution. ${ }^{18}$ The age and sex distribution of the population within the GPRD is also representative of the general UK population. It contains biographic data on all patients; information on any major illness or procedures; all drug prescription including dose, strength, formulation, indication, adverse reactions and coprescriptions, and details of consultation and referrals. The quality and completeness of information in the database has been shown to be valid by a number of external studies. ${ }^{19-21}$ Information from the database is completely anonymous. Ethical approval for this study was given by the Scientific and Ethical Advisory Group (SEAG) of the General Practice Research Database.

The source population for this study was 1441782 people registered with the 177 general practices actively contributing to the GPRD in June 2001. Cases were defined as women aged 45 years and over who were diagnosed for the first time with any type of cataract. The diagnosis of cataract was confirmed by hospital consultant ophthalmologists and the information forwarded to the general practitioner who records this in the medical records. ${ }^{22}$ The date of diagnosis was referred to as the index date. Cases were required to have at least 180 days of observation prior to the index date. A simple random sample of 10000 cases was selected from the source population. For each case, one control with no record of cataract anywhere in their medical records was selected. Controls were alive and registered with the same practice on the index date of their matched case, and were also matched to cases on age (within 1 year). Controls were assigned a pseudoindex date to match the index date of their matched case, and were also required to have at least 180 days of observation prior to this date. Observation periods were truncated if necessary to ensure that within each case-control set, the duration of observation period was the same.

Only drug exposures prior to the index date were included in the analyses. The specific constituents of each proprietary formulation of HRT were defined according to the British National Formulary. ${ }^{23}$ For our analysis, we examined exposure to oestrogen-only and oestrogenprogestogen HRT separately. Both HRT types were initially defined as binary variables - ever or never use. Ever use was also recategorised as past use (participants with prescriptions greater than 180 days prior to the index date but with no current prescriptions) and 'past and current' use (participants currently on HRT and with past prescriptions). Data on current use (participants with HRT prescription only within 180 days of the index date) alone was not used, as the numbers in this category were too small for meaningful comparisons.

Data on the following a priori confounders were also extracted from the database-diabetes, hypertension, glaucoma, systemic steroids, aspirin, body mass index (weight in kilograms divided by the square of height in metres), alcohol and smoking. The patient-doctor consultation rate was also considered a potential confounder because the frequency of consultation may influence the likelihood of both cataract diagnosis and drug prescription, and this variable has been shown to be an important confounder in previous studies using this database. ${ }^{24}$ The mean annual consultation rate for each participant was calculated by dividing the total number of consultations by the years of observation prior to the index date.

The appropriateness of matching the cases and controls by age was assessed using the mean age difference within case-control matched sets, calculated by summing the age difference within each case-control set and dividing this by the total number of sets.

Following this initial descriptive analysis, HRT use was modelled as a binary ever/never exposure using conditional logistic regression, examining oestrogen-only and oestrogen-progestogen combinations separately. We then fitted a series of bivariate models for each HRT type, retaining only the variables that changed the odds ratio for HRT by $10 \%$ or more in the models. All analyses were conducted using the STATA 7.0 software. ${ }^{25}$

The prevalence of HRT use among controls in this study population was $5.5 \%$. The study thus had $90 \%$ power at the $5 \%$ significance level to detect a reduction of 0.8 in the odds of developing cataract with HRT use.

\section{Results}

The characteristics of cases and controls are shown in Table 1 . The mean age of the participants was 81 years. The mean age difference within case-control sets was 0.01 year (standard deviation 0.12), suggesting that the matching on age was successful. The mean observation period was 4.4 years. In all, 35.8\% of cases consulted their doctors eight or more times per year compared to only $23.1 \%$ of controls. The higher the consultation rate, the higher an individual's risk of being diagnosed with cataract (Table 1)

Table 2 shows the univariate and adjusted association between cataract and HRT. In all, $6.04 \%$ of the cases had ever been exposed to oestrogen-only HRT, with $2.71 \%$ (271 of 604) classified as past users and 3.14\% (314 of 604) classified as 'past and current' users. The remaining 19 women were current users only, and therefore not included in the stratified analyses. A smaller percentage of controls were ever-users (5.50\%), with $2.11 \%$ (211 of 550) classified as past users and 3.07\% (307 of 550) classified as 'past and current users'. In all, 32 women were current users only. The proportion of cases and 
Table 1 Characteristics of cases and controls and univariate associations

\begin{tabular}{|c|c|c|c|c|}
\hline & Cases $(\mathrm{N}=10000)$ & Controls $(\mathrm{N}=10000)$ & Odds ratio $(95 \% \mathrm{CI})$ & P-value \\
\hline Mean age difference by set (year) (SD) & \multicolumn{2}{|c|}{$0.01(0.12)$} & & \\
\hline Mean age (years) $(S D)^{a}$ & $81(10.0)$ & $81(10.0)$ & & \\
\hline Mean observation period (years) (SD) & $4.4(2.6)$ & $4.4(2.6)$ & & \\
\hline \multicolumn{5}{|l|}{ Age (years) $)^{a}$} \\
\hline $45-74$ & $2254(22.5 \%)$ & $2254(22.5 \%)$ & & \\
\hline $75-84$ & $3659(36.6 \%)$ & $3662(36.6 \%)$ & & \\
\hline $85-89$ & $2065(20.7 \%)$ & $2066(20.7 \%)$ & & \\
\hline$\geq 90$ & $2022(20.2 \%)$ & $2020(20.2 \%)$ & & \\
\hline \multicolumn{5}{|l|}{ Mean annual consultation rate } \\
\hline$<2$ & $1066(10.7 \%)$ & $2872(28.7 \%)$ & Baseline & \\
\hline $2-4$ & $2773(27.7 \%)$ & $2802(28.0 \%)$ & $3.05(2.76-3.36)$ & \\
\hline $5-7$ & $2585(25.9 \%)$ & $2014(20.1 \%)$ & $4.17(3.76-4.63)$ & $<0.001^{*}$ \\
\hline$\geq 8$ & $3576(35.8 \%)$ & $2312(23.1 \%)$ & $5.30(4.78-5.87)$ & \\
\hline Diabetes & $1217(12.2 \%)$ & $626(6.30 \%)$ & $2.10(1.90-2.33)$ & $<0.001$ \\
\hline Hypertension & $4360(43.6 \%)$ & $3594(35.9 \%)$ & $1.39(1.31-1.48)$ & $<0.001$ \\
\hline Glaucoma & $1013(10.1 \%)$ & $498(4.98 \%)$ & $2.18(1.95-2.45)$ & $<0.001$ \\
\hline Systemic steroids & $1538(15.4 \%)$ & $1064(10.6 \%)$ & $1.56(1.43-1.70)$ & $<0.001$ \\
\hline Aspirin & $1996(20.0 \%)$ & $1517(15.2 \%)$ & $1.43(1.33-1.55)$ & $<0.001$ \\
\hline \multicolumn{5}{|l|}{ Body mass index $\left(\mathrm{kg} / \mathrm{m}^{2}\right)$} \\
\hline $15-21$ & $1550(15.5 \%)$ & $1228(12.3 \%)$ & Baseline & \\
\hline $22-24$ & $1856(18.6 \%)$ & $1568(15.7 \%)$ & $0.95(0.86-1.05)$ & 0.33 \\
\hline $25-27$ & $1635(16.4 \%)$ & $1382(13.8 \%)$ & $0.96(0.86-1.06)$ & 0.39 \\
\hline$\geq 28$ & $1913(19.1 \%)$ & $1616(16.2 \%)$ & $0.96(0.87-1.07)$ & 0.49 \\
\hline Missing data & $3046(30.5 \%)$ & $4206(42.1 \%)$ & & \\
\hline \multicolumn{5}{|l|}{ Alcohol consumption } \\
\hline Nondrinker & $2625(26.3 \%)$ & $2253(22.5 \%)$ & Baseline & \\
\hline Exdrinker & $50(0.50 \%)$ & $45(0.45 \%)$ & $0.95(0.63-1.45)$ & 0.82 \\
\hline Current drinker & $4939(49.4 \%)$ & $4226(42.3 \%)$ & $1.02(0.95-1.10)$ & 0.59 \\
\hline Missing data & $2386(23.9 \%)$ & $3476(34.8 \%)$ & & \\
\hline \multicolumn{5}{|l|}{ Smoking } \\
\hline Nonsmoker & $6461(64.6 \%)$ & $5440(54.4 \%)$ & Baseline & \\
\hline Exsmoker & $967(9.67 \%)$ & $798(7.98 \%)$ & $1.03(0.93-1.14)$ & 0.58 \\
\hline Current smoker & $1176(11.8 \%)$ & $1095(11.0 \%)$ & $0.93(0.85-1.02)$ & 0.14 \\
\hline Missing data & $1396(14.0 \%)$ & $2667(26.7 \%)$ & & \\
\hline
\end{tabular}

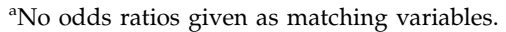

${ }^{*} P$-value for trend.

Table 2 Association between cataract and HRT - adjusted for mean annual consultation rate

\begin{tabular}{|c|c|c|c|c|c|}
\hline & $\begin{array}{c}\text { Cases } \\
(\mathrm{N}=10000)\end{array}$ & $\begin{array}{c}\text { Controls } \\
(\mathrm{N}=10000)\end{array}$ & $\begin{array}{l}\text { Univariate OR } \\
\qquad(95 \% \mathrm{CI})\end{array}$ & $\begin{array}{c}\text { Adjusted OR for consultation } \\
\text { rate }(95 \% \mathrm{CI})\end{array}$ & P-value \\
\hline \multicolumn{6}{|l|}{ Oestrogen-only } \\
\hline Never & $9396(94.0 \%)$ & $9450(94.5 \%)$ & Baseline & Baseline & \\
\hline Ever & $604(6.04 \%)$ & $550(5.50 \%)$ & $1.13(0.99-1.29)$ & $0.81(0.71-0.94)$ & $<0.001$ \\
\hline Past only & $271(2.71 \%)$ & $211(2.11 \%)$ & $1.33(1.10-1.61)$ & $0.92(0.75-1.12)$ & 0.41 \\
\hline Past and current & $314(3.14 \%)$ & $307(3.07 \%)$ & $1.05(0.89-1.26)$ & $0.78(0.65-0.94)$ & 0.009 \\
\hline \multicolumn{6}{|l|}{ Oestrogen-progestogen } \\
\hline Never & $9572(95.7 \%)$ & $9622(96.2 \%)$ & Baseline & Baseline & \\
\hline Ever & $428(4.28 \%)$ & $378(3.78 \%)$ & $1.18(1.01-1.39)$ & $0.86(0.72-1.02)$ & $<0.001$ \\
\hline Past only & $233(2.33 \%)$ & $166(1.66 \%)$ & $1.49(1.19-1.84)$ & $1.08(0.86-1.37)$ & 0.51 \\
\hline Past and current & $182(1.82 \%)$ & $190(1.90 \%)$ & $0.99(0.79-1.24)$ & $0.73(0.57-0.92)$ & 0.008 \\
\hline
\end{tabular}

controls ever exposed to oestrogen-progestogen HRT were 4.28 and $3.78 \%$, respectively. A total of 31 cases and 22 controls had been exposed to both oestrogen-only HRT and to oestrogen-progestogen HRT. The numbers were too small to analyse a mixed pattern of exposure on its own, and these women were included in the oestrogen-progestogen HRT group in the analysis. In a reanalysis excluding women exposed to both 
oestrogen-only and oestrogen-progestogen HRT, the results obtained were almost identical with the odds ratios differing by less than the second decimal place.

In the unadjusted analysis, oestrogen-only HRT users had a slightly increased risk of developing cataract compared to the never users (odds ratio 1.13, 95\% CI 0.99-1.29). While there was a significant increase in risk among the past users (odds ratio 1.33, 95\% CI 1.10-1.61), 'past and current' use was associated with a small nonsignificant increased risk of cataract (odds ratio 1.05, 95\% CI 0.89-1.26). The crude odds ratio for the association between cataract and any oestrogenprogestogen HRT exposure was 1.18 (95\% CI 1.01-1.39). The risk was higher among past users, such that they were about 1.5 times more likely to develop cataract than never users (odds ratio 1.49, 95\% CI 1.19-1.84). 'Past and current' use of this treatment was not associated with the risk of cataract in the crude analysis (odds ratio $0.99,95 \%$ CI 0.79-1.24).

The association between any HRT exposure and cataract changed substantially after adjusting for mean annual consultation rate, with a significantly lower risk of developing cataract in almost every group of HRT users. Among ever-users of oestrogen-only HRT, the odds ratio for cataract adjusted for consultation rate was 0.81 (95\% CI 0.71-0.94) compared with never-users; while among oestrogen-progestogen HRT users, the consultation rate-adjusted odds ratio for cataract was 0.86 (95\% CI 0.72-1.02). While past use of oestrogen-only HRT following adjustment for consultation rate reduced the risk of cataract (odds ratio 0.92, 95\% CI 0.75-1.12), past use of oestrogen-progestogen HRT was associated with a small increased risk of cataract (odds ratio 1.08, 95\% CI 0.86-1.37), although neither of these associations were statistically significant. 'Past and current' users of either formulation had a significant reduced risk of cataract after adjustment for consultation rate. The associations were not materially affected by adjusting for diabetes, hypertension, glaucoma, systemic steroids, or aspirin in the total sample or by adjusting for body mass index, alcohol or smoking.

\section{Discussion}

The results from this study suggest that use of oestrogenonly or oestrogen-progestogen HRT is associated with a small protective effect against cataract development once we adjusted for a measure of how often patients were seen by their GP. When patterns of use were examined, this effect seemed limited to 'past and current' users with no significant effects in women who had only used HRT in the past.
While the data from the GPRD are known to be relatively accurate and complete, ${ }^{19-21}$ alternative explanations for the results need to be considered. Selection bias is unlikely in this study, as cases and controls were randomly selected from the GPRD cohort of patients. There is also little potential for misclassification of the exposure, as all information was obtained from the database and not from individual patients, thus reducing the possibility of recall bias. A further strength of this study was that it was possible to determine the temporal sequence of events as drug use is known to have preceded the determination of disease status. The fact that HRT was prescribed does not, however, imply actual use. This may lead to some misclassification with regards to users and nonusers, although this is not likely to be differential between cases and controls. Validation studies comparing self-reported HRT use and information from general practitioners' records have also found good agreement (96\%). ${ }^{26}$ It was also not possible to study the cumulative effect of HRT use, as there was no information on its use before patients registered with the GPRD. Thus, incomplete ascertainment of past use may be greater in past users as opposed to current users and this may explain why we found no effect of past use on its own. It would be useful for future studies to look more closely at patterns of use and for a possible dose-response effect in order to fully understand the association.

There may have been more opportunity for misclassification of the outcome, particularly as systemic differences in ascertaining case status may exist between general practices in relation to the hospitals consulted. However, the validity of a recorded diagnosis of cataract in the GPRD has been found to be high $(94 \%)^{22}$ and we were able to ensure some uniformity in diagnostic pattern by matching cases and controls on the general practice used. In spite of this, one of the major challenges facing epidemiological research of cataract is the problem of case ascertainment. Cataract progresses from subclinical lesions that can only be detected by slit-lamp examination to lesions large enough and dense enough to obscure vision. It is possible that some people with smaller opacities may have been labelled as noncases. However, there is no reason to suspect this misclassification would be differential with regard to exposure status, meaning only that the effects under study may have been somewhat underestimated. In addition, information on the different cataract subtypes was not available. While it has been said that the effect of HRT may differ for the different subtypes of cataract, results from previous studies have been inconsistent. In this large, multiethnic database, all the cataract subtypes would have been represented. More importantly, the burden on the individual and health services is the 
presence of any cataract and not necessarily the cataract subtype.

In observational studies, women who take HRT tend to be generally healthier than women who do not. They are also more likely to adhere to other protective types of behaviour than nonusers. Failure to adjust for this potential confounding effect may lead to an overestimate of the protective effect of HRT. However, we were able to adjust for several indicators of health status in the multivariate analyses, but only mean annual consultation rate changed the crude effect of HRT on cataract. By matching on practice, we may have partially controlled for the effect of socioeconomic status (there is no information on this available in the GPRD) and there remains the possibility of uncontrolled confounding by unknown factors.

The levels of missing data for body mass index, alcohol consumption, and smoking were higher among controls than among cases. For both cases and controls, well over half the people with missing data were in the lowest category for consultation rate. This is as expected. People who attended the general practice less were less likely to have their body mass index, alcohol, and smoking status recorded. Controls were more likely to be in the lowest category for consultation rate, and were therefore more likely to have missing data from body mass index, alcohol, and smoking. The observed lack of association between cataract and current or exsmoking is inconsistent with smoking being an established risk factor for the disease. ${ }^{27}$ The crude odds ratio for the association between cataract and current smoking was 0.93 (95\% CI 0.85-1.02). While the prevalence of current smoking is consistent with that recorded in a large representative household survey in the UK, the level of exsmoking is much lower than would be expected..$^{28} \mathrm{We}$ have previously shown that for people recorded as being current smokers, the magnitude and direction of the dose-response relationship with the risk of lung cancer are what would be expected, ${ }^{29}$ suggesting that a code for current smoking is accurate. A likely explanation for the observed lack of association between cataract and either current or exsmoking when compared with nonsmoking is that many people recorded as being nonsmokers are in fact exsmokers.

Our results are mostly consistent with other previous studies of this association. For example, the protective effect of oestrogen-only HRT against any cataract found in this study agrees with results from the Nutrition and Vision Project in the United States. ${ }^{15}$ In addition, both Blue Mountains Eye Studies ${ }^{16,17}$ found that oestrogenprogestogen HRT had a protective effect against cortical and nuclear cataract. The only previous study in the $\mathrm{UK}^{13}$ a prospective cohort study based on a population of 250 participants, did not find any association with
HRT. Our sample was much larger than theirs however, and we may have been able to detect smaller effects than they could.

Evidence of biological plausibility of a protective effect of HRT is provided by several studies. First, oestrogen receptor messenger RNA has been found in the human lens, suggesting that oestrogen has a direct effect on the lens. ${ }^{30}$ In laboratory rat models, oestrogen has been found to protect against cataract induced by transforming growth factor- beta ${ }^{6}$ and by methylnitrosourea. ${ }^{7}$ Significantly, oestrogen has also been shown to have antioxidant properties. ${ }^{31}$ This is important in the context of this study as peroxidation within the lens may be one of the mechanisms leading to cataract formation. In addition, higher levels of reduced glutathione and gluthathione peroxidase (measures of antioxidant activity) have been found in postmenopausal women using HRT. ${ }^{32}$ Long-term use of tamoxifene citrate, an antioestrogen, has been associated with an increased risk of cataract. ${ }^{33}$

We have found that hormone replacement therapy has a protective, albeit weak, effect against cataract development. Our study also suggests that progestogen may not be involved in cataract aetiology, because the risk of cataract was similar for both HRT types. It therefore adds to the documented beneficial effects of HRT although, given the recent evidence of its effects on the development of breast cancer and cardiovascular disease, all potential benefits and risks of this therapy should be taken into account when considering its use.

\section{Acknowledgements}

We would like to thank Chris Smith and Magdy Boulis for help with data processing. The study was funded by the Gift of Thomas Pocklington (London, UK). Liam Smeeth is supported by a MRC clinical scientist fellowship (London, UK). Richard Hubbard is supported by a Wellcome Trust advanced fellowship (London, UK). Lisa Hurt is supported by a Wellcome Trust postdoctoral training fellowship (London, UK).

\section{References}

1 Beral V, Banks E, Reeves G, Appleby P. Use of HRT and the subsequent risk of cancer. J Epidemiol Biostat 1999; 4: 191-215.

2 Writing Group for the Women's Health Initiative Investigators. Risks and benefits of oestrogen plus progestin in healthy postmenopausal women: principal results from the Women's Health Initiative randomized controlled trial. JAMA 2002; 288: 321-333.

3 Million Women Study Collaborators. Breast cancer and hormone replacement therapy in the Million Women Study. Lancet 2003; 362: 419-427. 
4 Abraham S, Perz J, Clarkson R, Llewellyn-Jones D. Australian women's perceptions of hormone replacement therapy over 10 years. Maturitas 1995; 21: 91-95.

5 Torgerson DJ, Bell-Syer SE. Hormone replacement therapy and prevention of nonvertebral fractures: a meta-analysis of randomized trials. JAMA 2001; 285: 2891-2897.

6 Hales AM, Chamberlain CG, Murphy CR, McAvoy JW. Estrogen protects lenses against cataract induced by transforming growth factor-beta (TGF beta). J Exp Med 1997; 185: 273-280.

7 Bigsby RM, Cardenas H, Caperell-Grant A, Grubbs CJ. Protective effects of estrogen in a rat model of age-related cataracts. Proc Natl Acad Sci USA 1999; 96: 9328-9332.

8 Dolin P. Epidemiology of cataract. In: Johnson GJ, Minassian DC, Weale R (eds). The Epidemiology of Eye Disease. Chapman and Hall: London, 1998, pp 103-118.

9 World Health Organization Office of Information. Global Initiative for the Elimination of Avoidable Blindness. An Informal Consultation. WHO: Geneva, 1997 (unpublished document WHO/PBL/97.61).

10 Klein BEK. Lens opacities in women in Beaver Dam, Wisconsin: is there evidence of an effect of sex hormones? Trans Am Ophthalmol Soc 1993; 91: 517-544.

11 Freeman EE, Munoz B, Schein OD, West SK. Hormone replacement therapy and lens opacities: The Salisbury Eye Evaluation Project. Arch Ophthalmol 2001; 119: 1687-1692.

12 Worzala K, Hiller R, Sperduto RD, Mutalik K, Murabito JM, Moskowitz $\mathrm{M}$ et al. Postmenopausal estrogen use, type of menopause and lens opacities; The Framingham Studies. Arch Intern Med 2001; 161: 1448-1454.

13 Thompson JR, Deane JS, Hall AB, Rosenthal AR. Oestrogen and lens opacities in the Melton Eye Study (abstract). Invest Ophthalmol Vis Sci 1996; 37: S585.

14 Klein BE, Klein R, Lee KE. Reproductive exposures, incident age-related cataracts and age-related maculopathy in women: The Beaver Dam Eye Study. Am J Ophthalmol 2000; 130: 322-326.

15 Weintraub JM, Taylor A, Jacques P, Willett WC, Rosner B, Colditz GA et al. Postmenopausal hormone use and lens opacities. Ophthalmic Epidemiol 2002; 9: 179-190.

16 Cumming RG, Mitchell P. Hormone replacement therapy, reproductive factors and cataract. The Blue Mountains Eye Study. Am J Epidemiol 1997; 145: 242-249.

17 Younan C, Mitchell P, Cumming RG, Panchapakesan J, Rochtchina E, Hales AM. Hormone replacement therapy, reproductive factors and the incidence of cataract and cataract surgery. The Blue Mountains Eye Study. Am J Epidemiol 2002; 155: 997-1006.
18 Office for National Statistics. Key health Statistics from general practice 1996, Series MB6 No. 1. Office for National Statistics: London, 1998.

19 Nazareth I, King M, Haines A, Rangel L, Myers S. Accuracy of diagnosis of psychosis on general practice computer system. BMJ 1993; 307: 32-34.

20 Hollowell J. The General Practice Research Database: quality of morbidity data. Pop Trend 1997; 87: 36-40.

21 Office for National Statistics. Morbidity Statistics from General Practice-fourth national study 1991-92, Series MB5 No. 3. Office for National statistics: London, 1995.

22 Ruigomez A, Garcia Rodriguez LA, Dev VJ, Arellano F, Raniwala J. Are schizophrenia or antipsychotic drugs a risk factor for cataract? Epidemiology 2000; 11: 620-623.

23 British Medical Association and the Royal Pharmaceutical Society of Great Britain. British National Formulary, Vol. 44. Pharmaceutical Press: London, 2002.

24 Smeeth L, Hubbard R, Fletcher AE. A population based case-control study of cataract and inhaled corticosteroids. Br J Ophthalmol 2003; 000: 1-5.

25 STATA 7.0. Texas, Stata Corporation, 2001.

26 Banks E, Beral V, Cameron R, Hogg A, Langley N, Barnes I et al. Agreement between general practice prescription data and self-reported use of hormone replacement therapy and treatment for various illnesses. J Epidemiol Biostat 2001; 6: 357-363.

27 Christen WG, Glynn RJ, Ajani UA, Schaumberg DA, Buring JE, Hennekens $\mathrm{CH}$ et al. Smoking cessation and risk of age-related cataract in men. JAMA 2000; 284: 713-716.

28 Office for National Statistics. Living in Britain: preliminary results from the 1996 General Household Survey. The Stationery Office: London, 1997.

29 Hubbard R, Venn A, Lewis S, Britton J. Lung cancer and cryptogenic fibrosing alveolitis. A population-based cohort study. Am J Respir Crit Care Med 2000; 161: 5-8.

30 Ogueta SB, Schwartz SD, Yamashita CK, Farber DB. Estrogen receptor in the human eye: influence of gender and age on gene expression. Invest Ophthalmol Vis Sci 1999; 40: 1906-1911.

31 Mooradian AD. Antioxidant properties of steroids. J Steroid Biochem Mol Biol 1993; 45: 509-511.

32 Ozden S, Dildar K, Kadir YH, Gulizar K. The effects of hormone replacement therapy on lipid peroxidation and antioxidant status. Maturitas 2001; 38: 165-170.

33 Paganini-Hill A, Clark LJ. Eye problems in breast cancer patients treated with tamoxifen. Breast Cancer Res Treat 2000; 60: 167-172. 